\title{
Computation of springing transfer functions
}

\author{
H Söding* \\ Technical University Hamburg-Harburg, Hamburg, Germany
}

The manuscript was received on 12 February 2009 and was accepted after revision for publication on 27 April 2009.

DOI: 10.1243/14750902JEME158

\begin{abstract}
Ship vibrations excited continually by regular waves are predicted by a potential method. It takes into account the interaction of the oscillatory flow with the steady flow due to the ship's forward speed, including steady ship waves and squat, but it excludes non-linear oscillatory wave forces. A three-dimensional Rankine source patch method is used both for the steady and the oscillatory flow. Vibration damping by an immersed transom, wave radiation, bilge keels, propeller, and hatch cover friction are approximated. Application to a large containership demonstrates that, in this example, damping is mostly attributable to the transom.
\end{abstract}

Keywords: springing, hull vibration, seaway, transfer function, Rankine source method

\section{INTRODUCTION}

Springing designates continually excited vibrations of the total ship hull induced by the seaway. Whipping (slam-induced) vibrations and local (plate and panel) vibrations are not treated here. The paper is further restricted to excitations and responses depending linearly on wave height, in spite of the fact that in some ships, especially those with strongly flared sections, non-linear excitations are often predominant. Only transfer functions, i.e. springing amplitudes in regular waves of unit amplitude, are considered. These allow the significant amplitude of springing in natural seaways to be predicted according to the usual practice for any linear response.

The ship hull structure is modelled as a straight Timoschenko beam discretized by finite elements (FE). Thus, bending and shear stiffness are taken into account, but warping and local deformations are excluded. This appears appropriate for the lower modes of vertical bending; for horizontal bending this is appropriate only for ships with small or no hatch openings.

The correct determination of the excitation is both important and difficult. Older methods, e.g. those

*Corresponding author: Department of Fluid Dynamics and Ship Theory, Hamburg University of Technology, Institute M-8, Raum 5058, Schwarzenbergstrasse 95 C, Hamburg, D-21073, Germany. email: h.soeding@tu-harburg.de given in references [1] and [2], and even several modern ones use adaptations of the strip method to springing. Because substantial linear springing responses are excited by short waves only, the excitation cannot accurately be determined from the two-dimensional (2D) flow around ship sections in planes $x=$ constant, as is typical for the strip method. Instead, Newman's 'reverse flow theorem' [3] was sometimes used. However, this mathematically elegant method is not directly applicable to ships with an immersed transom, and the correction proposed for this case in reference [2] appears questionable.

Today it seems appropriate to use either threedimensional (3D) potential flow methods or RANS methods [4] to predict springing. The latter appear good for highly involved investigations and for verifying other methods, but too slow for routine application. Therefore a potential flow method is described here. It uses Rankine sources, which do not automatically satisfy the free surface boundary condition. Instead, the high-frequency limit $\phi=0$ of the exact free surface boundary condition is satisfied here by using a mirror sink above the water surface for each original source below the water surface. The patch method [5] is used to discretize the source distribution.

Interactions between the regular, periodical waves, and the stationary (in a ship-fixed reference system) waves owing to forward speed are taken into 
account both for excitation and motion-dependent pressures. Especially the stern wave may have substantial influence on eigenfrequencies, excitation, and damping. Therefore the steady flow problem is solved first, satisfying the steady, fully non-linear free-surface boundary conditions. Whereas for the steady flow a panel mesh both on the wetted hull and on part of the free surface is used, the periodical flow requires only panels on the hull. For both flows the same set of triangular hull panels is used.

The free-surface condition $\phi=0$ eliminates damping by wave radiation. Thus without adding empirical damping the response would be excessive at resonance. To correct that, damping by various processes is discussed, and approximate formulae for it are developed.

\section{MOTION EQUATION}

A coordinate system $x, y, z$ with axes directed forward, to starboard and downward, respectively, is used. Its origin is at the intersection of the midship section, the symmetry plane, and the undisturbed water surface.

Only harmonic vibrations of frequency $\omega_{\mathrm{e}}$, the encounter frequency between ship and waves, are considered

$$
\vec{u}(x, t)=\operatorname{Re}\left(\hat{\vec{u}}(x) \mathrm{e}^{i \omega_{\mathrm{e}} t}\right)
$$

Here and in the following the hat symbol (as in $\hat{\vec{u}}$ ) designates a complex amplitude. Its real part is the value at the instant when a wave trough is at the coordinate origin, and the imaginary part is the value one quarter period earlier (if $\omega_{\mathrm{e}}>0$ ) or later (if $\omega_{\mathrm{e}}<0$ ).

The motion equation of the body may be written in the form

$$
\mathcal{K}[\vec{u}(x)]+\mathcal{D}[\dot{\vec{u}}(x)]+\mathcal{M}[\ddot{\vec{u}}(x)]=\overrightarrow{\mathcal{F}}(x)
$$

$\mathcal{K}$ is the stiffness operator, i.e. the outer force distribution required to produce the deformation $\vec{u}(x)$ if the body had only stiffness, but no damping and mass. Correspondingly $\mathcal{D}$ and $\mathcal{M}$ are the damping and mass operators, respectively. $\overrightarrow{\mathcal{F}}(x)$ is the distribution of excitation forces and moments caused, here, by the periodical part of the pressure distribution on the wetted hull.
Inserting equation (1) into equation (2) and using the fact that $\mathcal{K}, \mathcal{D}$, and $\mathcal{M}$ are linear operators results in the equation

$$
\underbrace{\left(\mathcal{K}+i \omega_{\mathrm{e}} \mathcal{D}-\omega_{\mathrm{e}}^{2} \mathcal{M}\right)}_{-\mathcal{V}} \hat{\vec{u}}(x)=\hat{\overrightarrow{\mathcal{F}}}(x)
$$

thus

$$
\mathcal{V}[\hat{\vec{u}}(x)]+\hat{\overrightarrow{\mathcal{F}}}(x)=0
$$

When it is not convenient to separate the motiondependent forces into stiffness, damping, and mass contributions, the operator $\mathcal{V}$ (for vibration-dependent force matrix) will be used. $\mathcal{V}$ comprises contributions from the structural stiffness and the ship's mass $\left(\mathcal{V}_{S}\right)$, the added mass effect of the surrounding water $\left(\mathcal{V}_{p}\right)$, the weight distribution $\left(\mathcal{V}_{W}\right)$, and various damping effects.

\section{FE DISCRETIZATION}

The continuous function $\hat{\vec{u}}(x)$ is represented by a vector $\hat{\boldsymbol{u}}$. Its components are the translations in $y$ and $z$ direction and the rotations of the crosssections around the $x, y$, and $z$ axes at all FE nodes of the Timoschenko beam. Longitudinal vibrations are omitted because their eigenfrequencies are quite high, and because their coupling with the transverse and vertical bending is small.

The equation of motion (3) can be transformed into the FE equation

$$
\left(\mathbf{K}+i \omega_{\mathrm{e}} \mathbf{D}-\omega_{\mathrm{e}}^{2} \mathbf{M}\right) \hat{\boldsymbol{u}}=\hat{\boldsymbol{F}}
$$

where $\mathbf{K}, \mathbf{D}$, and $\mathbf{M}$ are the stiffness, damping, and mass matrices, respectively, and $\hat{\boldsymbol{F}}$ is the excitation vector.

\section{USE OF APPROXIMATE MODES}

The operators $\mathcal{K}, \mathcal{D}$, and $\mathcal{M}$, and the FE matrices $\mathbf{K}$, $\mathbf{D}$, and $\mathbf{M}$ as well, depend on the mode shape. For example, in $\mathbf{K}$ the shear lag is responsible for a reduction of the bending stiffness $E I$ depending on the mode. Similarly, the added-mass contribution to $\mathbf{M}$ is smaller for higher modes than for lower ones. Thus, in the following the quantities $\mathbf{K}, \mathbf{D}$, and $\mathbf{M}$ are written with an index $j$ indicating a certain mode.

This is one reason for introducing mode vectors $\boldsymbol{w}_{j}$ from which the actual deformation $\boldsymbol{u}$ is superimposed

$$
\hat{\boldsymbol{u}}=\sum_{j=1}^{J} \boldsymbol{w}_{j} \hat{v}_{j}
$$


$J$ is the number of modes taken into account. $\boldsymbol{w}_{j}$ are real vectors designating the translations of the nodes and the rotations of the cross sections in mode $j . \hat{v}_{j}$ are complex scalars: the superposition factors of the different modes when solving the equation for forced vibrations in a wave.

Using equation (5), the motion equation (4) becomes

$$
\sum_{j=1}^{J}\left(\mathbf{K}_{j}+i \omega_{\mathrm{e}} \mathbf{D}_{j}-\omega_{\mathrm{e}}^{2} \mathbf{M}_{j}\right) \boldsymbol{w}_{j} \hat{v}_{j}=\hat{\boldsymbol{F}}
$$

If $J$ is smaller than the number of degrees of freedom of the FE system, this is an overdetermined system for the unknown amplitudes $\hat{v}_{j}$. To obtain a system of equations having as many equations as unknowns, and approximately to decouple the equations, equation (6) is multiplied from the left by $\boldsymbol{w}_{k}^{T}$, where $T$ indicates the transpose, and $k$ is a mode index $(1 \leqslant k \leqslant J)$

$$
\begin{aligned}
& \sum_{j=1}^{J}\left(\boldsymbol{w}_{k}^{T} \mathbf{K}_{j} \boldsymbol{w}_{j}+i \omega_{\mathrm{e}} \boldsymbol{w}_{k}^{T} \mathbf{D}_{j} \boldsymbol{w}_{j}-\omega_{\mathrm{e}}^{2} \boldsymbol{w}_{k}^{T} \mathbf{M}_{j} \boldsymbol{w}_{j}\right) \hat{v}_{j}=\boldsymbol{w}_{k}^{T} \hat{\boldsymbol{F}}, \\
& \quad k=1 \ldots J
\end{aligned}
$$

For fixed $k$ and $j$, the expression $\boldsymbol{w}_{k}^{T} \mathbf{K}_{j} \boldsymbol{w}_{j}$ is a real scalar: the virtual work produced by the stiffness forces $\mathbf{K}_{j} \boldsymbol{w}_{j}$ and the virtual deformations $\boldsymbol{w}_{k}$. For the different possible values of $k$ and $j$, these $J^{2}$ scalars are combined to the $J \times J$ matrix $K$

$$
K=\left(\boldsymbol{w}_{k}^{T} \mathbf{K}_{j} \boldsymbol{w}_{j}, \quad k=1 \ldots J, \quad j=1 \ldots J\right)
$$

Correspondingly for $D$ and $M$

$$
\begin{aligned}
& D=\left(\boldsymbol{w}_{k}^{T} \mathbf{D}_{j} \boldsymbol{w}_{j}, \quad k=1 \ldots J, \quad j=1 \ldots J\right) ; \\
& M=\left(\boldsymbol{w}_{k}^{T} \mathbf{M}_{j} \boldsymbol{w}_{j}, \quad k=1 \ldots J, \quad j=1 \ldots J\right)
\end{aligned}
$$

$\hat{F}$ is the column vector the components of which are $\boldsymbol{w}_{k}^{T} \hat{\boldsymbol{F}}$

$$
\hat{F}=\left(\boldsymbol{w}_{k}^{T} \hat{\boldsymbol{F}}, \quad k=1 \ldots J\right)
$$

This gives the following matrix equation

$$
\underbrace{\left(K+i \omega_{\mathrm{e}} D-\omega_{\mathrm{e}}^{2} M\right)}_{-V} \hat{v}=\hat{F}
$$

where the matrices are square $J \times J$ matrices, and the column vectors $\hat{v}$ and $\hat{F}$ have $J$ components.
If $\boldsymbol{w}_{j}$ were the exact mode shapes, and if $K$ and $M$ were the same for all modes, then $K$ and $M$ were diagonal matrices. However, the mode vectors and the matrices $K$ and $M$ cannot be determined independently. Thus an iteration is required to determine the exact mode shapes. To avoid this complication, here approximate mode vectors $\boldsymbol{w}_{j}$ are used. They are determined by an eigenvalue analysis from the equation for free, undamped vibrations

$$
\left(K-\omega_{\mathrm{e}}^{2} M\right) \boldsymbol{w}=0
$$

where $K$ and $M$ are estimations of the stiffness and mass matrix. $K$ is determined from the structural stiffness for the 2-node bending mode and the hydrostatical restoring force, and $M$ from the solid mass distribution together with added masses determined from 2D added masses and a reduction factor of 0.75 for $3 \mathrm{D}$ effects. These approximations are used only to determine $\boldsymbol{w}_{j}$; the springing vibrations are determined from the correct, $j$ dependent stiffness, damping and mass matrices as a superposition of the approximate mode shapes.

Without any supports the beam representing the ship structure may perform rigid-body motions as well as vibrations. Here the 'rigid-body motions' are included as low-frequency modes. They describe not exactly rigid motions, and their eigenfrequencies are not zero, owing to the effect of hydrostatic pressures, which contribute to the stiffness matrix. For vertical vibrations, the lowest two modes are, usually, not heave and pitch because both motions are coupled; instead the lowest two modes give two different superpositions of heave and pitch motions, including slight bending.

\section{CONTRIBUTION OF THE SHIP STRUCTURE AND OF SHIP MASS}

The current programme is restricted to vertical vibrations. For these, the structural part of the stiffness matrix is assembled from the following beam element stiffness matrices taken from reference [6]

$$
\begin{aligned}
K_{E}=\frac{E I}{(1+\kappa) l_{E}^{3}} & {\left[\left(\begin{array}{cccc}
12 & -6 l_{E} & -12 & -6 l_{E} \\
& 4 l_{E}^{2} & 6 l_{E} & 2 l_{E}^{2} \\
& & 12 & 6 l_{E} \\
& & & 4 l_{E}^{2}
\end{array}\right)\right.} \\
& \left.+\kappa\left(\begin{array}{cccc}
0 & 0 & 0 & 0 \\
& 1 l_{E}^{2} & 0 & -1 l_{E}^{2} \\
& & 0 & 0 \\
& & & 1 l_{E}^{2}
\end{array}\right)\right]
\end{aligned}
$$


with $E I$ designating bending stiffness, $G A_{\mathrm{s}}$ shear stiffness, $l_{E}$ element length and

$$
\kappa=\frac{12 E I}{G A_{s} l_{E}^{2}}
$$

(The lower-left part of the symmetrical matrices is omitted.)

Correspondingly the mass element matrix is

$$
\begin{aligned}
& M_{E}=\frac{1}{(1+\kappa)^{2}}\left[\frac{\mu l_{E}}{420}\left(\begin{array}{cccc}
156 & -22 l_{E} & 54 & 13 l_{E} \\
& 4 l_{E}^{2} & -13 l_{E} & -3 l_{E}^{2} \\
& & 156 & 22 l_{E} \\
& & & 4 l_{E}^{2}
\end{array}\right)\right. \\
& +\frac{\kappa \mu l_{E}}{120}\left(\begin{array}{cccc}
84 & -11 l_{E} & 36 & 9 l_{E} \\
& 2 l_{E}^{2} & -9 l_{E} & -2 l_{E}^{2} \\
& & 84 & 11 l_{E} \\
& & & 2 l_{E}^{2}
\end{array}\right) \\
& +\frac{\kappa^{2} \mu l_{E}}{120}\left(\begin{array}{cccc}
40 & -5 l_{E} & 20 & 5 l_{E} \\
& 1 l_{E}^{2} & -5 l_{E} & -1 l_{E}^{2} \\
& & 40 & 5 l_{E} \\
& & & 1 l_{E}^{2}
\end{array}\right) \\
& +\frac{\bar{\mu}}{30 l_{E}}\left(\begin{array}{cccc}
36 & -3 l_{E} & -36 & -3 l_{E} \\
& 4 l_{E}^{2} & 3 l_{E} & -1 l_{E}^{2} \\
& & 36 & 3 l_{E} \\
& & & 4 l_{E}^{2}
\end{array}\right) \\
& +\frac{\kappa \bar{\mu}}{6 l_{E}}\left(\begin{array}{cccc}
0 & 3 l_{E} & 0 & 3 l_{E} \\
& 1 l_{E}^{2} & -3 l_{E} & -1 l_{E}^{2} \\
& & 0 & -3 l_{E} \\
& & & 1 l_{E}^{2}
\end{array}\right) \\
& +\frac{\kappa^{2} \bar{\mu}}{6 l_{E}}\left(\begin{array}{cccc}
0 & 0 & 0 & 0 \\
& 2 l_{E}^{2} & 0 & 1 l_{E}^{2} \\
& & 0 & 0 \\
& & & 2 l_{E}^{2}
\end{array}\right)
\end{aligned}
$$

with $\mu=$ solid mass per length and $\bar{\mu}=$ solid mass moment of inertia around the $y$ axis per length. The above expressions result from the assumptions of constant mass density and stiffness within each finite element, and from a relation between crosssection rotation at the element ends and along the element, which is correct for statical deformations.

The total structural stiffness matrix $\mathbf{K}_{\mathrm{S}}$ and the total ship mass matrix $\mathbf{M}_{\mathrm{S}}$ are built from the above element matrices as usual in FE computations. From these matrices $K_{\mathrm{S}}$ and $M_{\mathrm{S}}$ are determined using equations (8) and (9) analogously.

\section{CONTRIBUTION OF THE SHIP'S WEIGHT}

For this small contribution the ship's mass is assumed to be the sum of point masses $m_{l}$ at locations $\vec{x}_{l}$. The weight forces $\vec{G}_{l}=\left(0,0, g m_{l}\right)$ are constant over time and, thus, have no influence on vibrations. But for a vibration of mode $j$ the moments of the weight force have an oscillatory part. For $\hat{v}_{j}=1$ it has the amplitude

$$
\vec{w}_{j} \times \vec{G}_{l}
$$

Together with the virtual motion of mode $k$ this moment produces the virtual work

$$
V_{W k j}=\sum_{l} \vec{\chi}_{k}\left(\vec{w}_{j} \times \vec{G}_{l}\right)=\sum_{l} \vec{G}_{l}\left(\vec{\chi}_{k} \times \vec{w}_{j}\right)
$$

where $\vec{\chi}_{k}$ designates the rotation angle around $x, y$, and $z$ axes of the beam cross-sections in mode $k$ at the location $\vec{x}_{l}$, and $\vec{w}_{j}$ is the translation of the shipfixed point $\vec{x}_{l}=(x, y, z)$ in mode $j$

$$
\vec{w}_{j}(\vec{x})=\vec{w}_{j}\left(\vec{x}_{0}\right)+\vec{\chi}_{j} \times\left(\vec{x}-\vec{x}_{0}\right)
$$

$z_{0}$ is the $z$ coordinate of the beam axis. $\vec{x}_{0}$ is the vector having components $x, 0, z_{0}$. At the FE nodes, $\vec{w}_{j}\left(\vec{x}_{0}\right)$ is equal to certain components of the node deformation vector $\boldsymbol{w}_{j}$; between the nodes, $\vec{w}_{j}\left(x, 0, z_{0}\right)$ and $\vec{\chi}_{k}$ are interpolated.

The second form in equation (17) is simpler to evaluate because only the $z$ component of $\vec{\chi}_{k} \times \vec{w}_{j}$ is required. Combining $V_{W k j}$ for all modes $k$ and $j$ to a matrix $V_{W}$ gives the weight contribution $V_{W}$ to $V$ in equation (11).

\section{CONTRIBUTION OF THE HULL PRESSURE}

The water around the ship generates a pressure distribution on the wetted part of the hull. In equation (11), the part of the pressure distribution due to the ship vibrations is represented by the contribution $V_{P}$ to $V$, whereas the part owing to the waves is represented by the excitation vector $\hat{F}$.

To determine $V_{P}$ and $\hat{F}$, the unit normal vector $\overrightarrow{\tilde{n}}$ on the hull is needed. $\overrightarrow{\tilde{n}}$ contains a stationary and an oscillatory part 


$$
\overrightarrow{\tilde{n}}=\vec{n}+\vec{\alpha} \times \vec{n}
$$

$\vec{n}$ is the normal vector for the non-oscillating ship. The tilt vector $\vec{\alpha}$ of the hull normal due to its oscillations is superimposed from the modes $j$

$$
\vec{\alpha}=\operatorname{Re}\left(\sum_{j} \vec{\alpha}_{j} \hat{v}_{j} \mathrm{e}^{i \omega_{\mathrm{e}} t}\right)
$$

The modal contributions $\vec{\alpha}_{j}$ differ from the modal contributions to the tilt vector of the cross sections, $\vec{\chi}_{j}$, owing to the shear deformation; for the most part of the ship a better approximation than $\vec{\chi}_{j}$ is

$$
\begin{aligned}
\vec{\alpha}_{j}= & \left(\chi_{j x},-w_{j z}^{\prime}, w_{j y}^{\prime}\right) \\
= & \left(\chi_{j x},-w_{j z}^{\prime}\left(x, 0, z_{0}\right)\right. \\
& \left.\quad-\chi_{j x}^{\prime} y, w_{j y}^{\prime}\left(x, 0, z_{0}\right)-\chi_{j x}^{\prime}\left(z-z_{0}\right)\right)
\end{aligned}
$$

Here the indices $x, y, \mathrm{z}$ designate vector components, and ' designates a (partial for $w_{j}$ ) derivative with respect to $x$. The last expression given in equation (21) results from introducing (18).

$V_{\mathrm{P}}$ is determined, corresponding to the equations (8) and (9), as the virtual work produced by:

(a) the complex amplitude of the oscillating pressure $\hat{p}_{j}(\vec{x})$ owing to the hull motion $\vec{w}_{j}(\vec{x})$, times;

(b) the hull motion $\vec{w}_{k}$ in the direction of the pressure force, i.e. normal to the hull

$$
\begin{aligned}
V_{P} & =\left(\int_{S} \vec{w}_{k}(\vec{x})\left[\hat{p}_{j}(\vec{x}) \vec{n}(\vec{x})+\left(\vec{\alpha}_{j}(\vec{x}) \times \vec{n}(\vec{x})\right) p^{(0)}\right] \mathrm{d} S\right. \\
k & =1 \ldots J, \quad j=1 \ldots J)
\end{aligned}
$$

where $\vec{x}$ designates points on the wetted hull surface $S$. The oscillatory change of this surface due to the exciting waves and due to springing need not be taken into account because its contribution depends quadratically on wave amplitude. $p^{(0)}$ is the stationary pressure at the hull, including the hydrostatic pressure, but excluding the air pressure because the latter acts also on the interior side of the hull.

The corresponding equation for the excitation vector is

$$
\hat{F}=\left(\int_{S} \vec{w}_{k}(\vec{x})\left(\hat{p}^{w}+\hat{p}^{d}\right) \vec{n}(\vec{x}) \mathrm{d} S, \quad k=1 \ldots j\right)
$$

Here $\hat{p}^{w}$ is the complex amplitude of the pressure owing to the wave, and $\hat{p}^{d}$ is the complex amplitude of the diffraction pressure, i.e. the change of the oscillatory pressure due to the presence of the nonvibrating ship

The pressure distributions $\hat{p}_{j}$ and $\hat{p}^{d}$ follow from the radiation potential $\phi_{j}$ and the diffraction potential $\phi^{d}$ of the water flow around the ship, respectively.

\section{DETERMINATION OF FLOW POTENTIALS}

The flow potentials are determined here by a 3D Rankine panel method. Because of the quadratical terms in the Bernoulli equation, interactions between the stationary and the oscillatory flow around the ship contribute to the oscillatory pressure even to first order. Thus it is necessary to have the total potential

$$
\phi^{t}=\phi^{(0)}+\underbrace{\operatorname{Re}\left(\hat{\phi}^{w}+\hat{\phi}^{d}+\sum_{j=1}^{J} \hat{\phi}^{j} \hat{v}_{j}\right) \mathrm{e}^{i \omega_{e} t}}_{\phi^{(1)}}
$$

$\hat{\phi}^{w}, \hat{\phi}^{d}$, and $\hat{\phi}^{j}$ are the complex amplitudes of the oscillatory potentials of the wave, the diffraction, and the radiation for unit motion of mode $j$, respectively. Determining $\hat{\phi}^{d}$ is the main difficulty in springing predictions. Emphasis on the accurate determination of $\hat{\phi}^{j}$, including, e.g. effects of the forward speed of the ship.

The potential of the steady flow around the ship is $\phi^{(0)}$. It is determined by the non-linear Rankine source method contained in the program GLRankine. The main features of this method are described in reference [7].

The wave potential $\hat{\phi}^{w}$ is well known. Its complex amplitude is (for deep water)

$$
\hat{\phi}^{w}=-i c e^{\vec{v} \cdot \vec{x}} \quad \text { with } \quad \vec{v}=i k(-\cos \mu, \sin \mu, i)
$$

Here $c=g / \omega$ is the wave celerity, $k=\omega^{2} / g$ the wave number, and $\mu$ is the wave direction ( 0 for following waves). $g$ is the gravity acceleration. Wave frequency $\omega$ and encounter frequency $\omega_{e}$ are related by

$$
\omega_{e}=\omega-k U \cos \mu
$$

where $U$ is ship speed.

For linear springing, substantial amplitudes occur only for wave encounter frequencies near to one of the vibration eigenfrequencies. For large ships, the lowest of these frequencies is, typically, around $0.5 \mathrm{~Hz}$. The wave length corresponding to this encounter frequency is too short to be resolved by 
a surface panel grid. Thus, for determining $\hat{\phi}^{d}$ and $\hat{\phi}^{j}$ the linearized boundary condition at the water surface

$$
g \hat{\phi}_{z}=\left(-U \frac{\partial}{\partial x}+i \omega_{e}\right)^{2} \hat{\phi}
$$

is substituted by its high-frequency limit

$$
\hat{\phi}=0
$$

In case of a plane water surface this condition is easily satisfied by arranging, above the water surface, mirror images of the sources below the water surface, and by giving each mirror source the negative source strength of the original source. However, here the free-surface boundary condition shall be satisfied at the water surface including steady ship waves. In this case mirror sources can satisfy the condition $\hat{\phi}=0$ only approximately. However, the error appears negligible if the mirror sources are arranged according to the local height of the waterline at the respective $x$ coordinate. Because the free-surface condition is satisfied in this way, albeit approximately, no panel grid on the free surface is required, in contrast to Rankine panel methods to determine steady ship waves or rigidbody motions in waves.

The source strengths are determined from the body boundary condition

$$
\overrightarrow{\tilde{n}}(\vec{x})[\vec{v}(\vec{x}+\vec{w})-\dot{\vec{w}}(\vec{x})]=0
$$

The condition expresses the fact that there is no flow through the hull; thus the normal component of the fluid flow speed $\vec{v}$ is equal to the normal component of the hull velocity $\dot{\vec{w}}$.

In equation (29), the argument $\vec{x}+\vec{w}$ of $\vec{v}$ takes account of the fact that, owing to the vibrations, the hull moves through the flow field represented by the potential $\phi^{t}$. If higher than first-order terms are omitted

$$
\vec{v}(\vec{x}+\vec{w})=\nabla \phi^{(0)}+(\vec{w} \nabla) \nabla \phi^{(0)}+\nabla \phi^{(1)}
$$

where all potentials are evaluated at point $\vec{x}$. Inserting this into equation (29) and using equations (19) and (20) gives the hull boundary condition in the form

$$
(\vec{n}+\vec{\alpha} \times \vec{n})\left[\nabla \phi^{(0)}+(\vec{w} \nabla) \nabla \phi^{(0)}+\nabla \phi^{(1)}-\dot{\vec{w}}(\vec{x})\right]=0
$$

The stationary term in this condition is zero due to the hull boundary condition of $\phi^{(0)}$. Keeping only the first-order oscillatory terms gives, after rearrangement of the first term

$$
\vec{w} \underbrace{(\vec{n} \nabla) \nabla \phi^{(0)}}_{\vec{m}}+\vec{n} \nabla \phi^{(1)}-\vec{n} \overrightarrow{\vec{w}}+(\vec{\alpha} \times \vec{n}) \nabla \phi^{(0)}=0
$$

where $\vec{m}$ is the so-called $m$-term. Inserting

$$
\vec{w}=\sum_{j=1}^{J} \vec{w}_{j}(\vec{x}) v_{j}(t)=\operatorname{Re}\left(\sum_{j=1}^{J} \vec{w}_{j}(\vec{x}) \hat{v}_{j} e^{i \omega_{\mathrm{e}} t}\right)
$$

and the expression given in equation (24) for $\phi^{(1)}$ gives a boundary condition for the complex amplitudes

$$
\begin{array}{r}
\vec{n}\left(\nabla \hat{\phi}^{w}+\nabla \hat{\phi}^{d}\right)+\sum_{j}\left[\vec{n} \nabla \hat{\phi}^{j}+\vec{w}_{j}\left(\vec{m}-i \omega_{e} \vec{n}\right)\right. \\
\left.+\left(\vec{\alpha}_{j} \times \vec{n}\right) \nabla \phi^{(0)}\right] \hat{v}_{j}=0
\end{array}
$$

The condition is satisfied for arbitrary $\hat{v}_{j}$ by using for the diffraction potential $\hat{\phi}^{d}$ the hull boundary condition

$$
\vec{n}\left(\nabla \hat{\phi}^{w}+\nabla \hat{\phi}^{d}\right)=0
$$

and for each of the mode radiation potentials $\hat{\phi}^{j}$ the condition

$$
\vec{n} \nabla \hat{\phi}^{j}+\vec{w}_{j}\left(\vec{m}-i \omega_{e} \vec{n}\right)+\left(\vec{\alpha}_{j} \times \vec{n}\right) \nabla \phi^{(0)}=0
$$

In reference [8] hull boundary conditions for rigid-body motions in waves are given. It can be shown that the conditions (35) and (36) result in these rigid-body conditions if one takes rigid-body motions as mode shapes.

\section{DETERMINATION OF THE PRESSURE AT POINTS MOVING WITH THE SHIP}

Consider a point at average position $\overrightarrow{\vec{x}}$. If the point moves with the hull, it has an actual position in the inertial reference frame of

$$
\vec{x}=\overrightarrow{\vec{x}}+\vec{w}
$$

At this hull-fixed point the difference between the fluid pressure and the air pressure is, according to Bernoulli's equation and up to first order

$$
\begin{aligned}
p(\vec{x})= & \frac{1}{2} \rho\left[U^{2}-\left(\nabla \phi^{(0)}\right)^{2}\right]-\rho \nabla \phi^{(0)} \nabla \phi^{(1)} \\
& -\rho \dot{\phi}^{(1)}+\rho g z+\vec{w} \nabla p(\overline{\vec{x}})
\end{aligned}
$$


where $\rho$ is fluid density. Elaborating the term $\nabla p$ gives, up to first order

$$
\begin{aligned}
p(\vec{x})= & \frac{1}{2} \rho\left[U^{2}-\left(\nabla \phi^{(0)}\right)^{2}\right]-\rho \nabla \phi^{(0)} \nabla \phi^{(1)} \\
& -\rho \dot{\phi}^{(1)}+\rho g z-\rho \vec{w}\left(\nabla \phi^{(0)} \nabla\right) \nabla \phi^{(0)}+\rho g w_{z}
\end{aligned}
$$

Separating the stationary pressure $p^{(0)}$ from the oscillatory pressure $p^{(1)}$ gives

$$
\begin{aligned}
p^{(0)}= & \frac{1}{2} \rho\left[U^{2}-\left(\nabla \phi^{(0)}\right)^{2}\right]+\rho g z \\
p^{(1)}= & -\rho \nabla \phi^{(0)} \nabla \phi^{(1)}-\rho \dot{\phi}^{(1)} \\
& -\rho \vec{w}\left(\nabla \phi^{(0)} \nabla\right) \nabla \phi^{(0)}+\rho g w_{z}
\end{aligned}
$$

From equation (41) follows the complex amplitude of the pressure

$$
\begin{aligned}
\hat{p}=\rho[ & -\nabla \phi^{(0)} \nabla \hat{\phi}^{(1)}-i \omega_{e} \hat{\phi}^{(1)} \\
& \left.-\hat{\vec{w}}\left(\nabla \phi^{(0)} \nabla\right) \nabla \phi^{(0)}+g \hat{w}_{z}\right]
\end{aligned}
$$

To obtain $\hat{p}^{w}+\hat{p}^{d}$ one has to insert $\hat{\phi}^{w}+\hat{\phi}^{d}$ for $\hat{\phi}^{(1)}$. This gives

$$
\hat{p}^{w}+\hat{p}^{d}=\rho\left[-\nabla \phi^{(0)}\left(\nabla \hat{\phi}^{w}+\nabla \hat{\phi}^{d}\right)-i \omega_{e}\left(\hat{\phi}^{w}+\hat{\phi}^{d}\right)\right]
$$

To obtain $\hat{p}_{j}$ one inserts $\hat{\phi}^{j} \hat{v}_{j}$ for $\hat{\phi}^{(1)}$. This gives

$$
\begin{aligned}
\hat{p}_{j}=\rho[ & -\nabla \phi^{(0)} \nabla \hat{\phi}^{j}-i \omega_{e} \hat{\phi}^{j} \\
& \left.-\hat{\vec{w}}_{j}\left(\nabla \phi^{(0)} \nabla\right) \nabla \phi^{(0)}+g \hat{w}_{j z}\right] \hat{v}_{j}
\end{aligned}
$$

$\hat{p}_{j}$ together with $p^{(0)}$ from (40) and $\hat{p}^{w}+\hat{p}^{d}$ are used in equations (22) and (23), respectively, to determine the pressure terms $V_{P}$ and $\vec{F}$.

\section{SOME NUMERICAL DETAILS}

The $m$-term in equation (32) and equation (44) contain second derivatives of the stationary potential $\phi^{(0)}$. In normal panel methods these terms cause difficulties because second derivatives do not converge (for decreasing panel dimensions) to the correct values due to irregularities of the flow caused by discontinuities of the source distributions. Here the patch method [5] is used. In this modification of the panel method one determines only averages of the second derivative terms over panels. These averages converge much better than the values at collocation points. Thus the panel averages of the $m$ terms can be computed directly from the potential and its normal derivative at the three vertices of each triangular panel. On the other hand, the panel averages of $\left(\nabla \phi^{(0)} \nabla\right) \nabla \phi^{(0)}$ in equation (44) are determined from the averaged first derivatives of $\phi^{(0)}$ at the three neighbour panels. Details of this procedure are explained in reference [8] for computing rigid-body motions. The same method proved applicable here.

Equation (25) for the linear wave potential also requires some comments: there is a superposition of steady ship waves with periodical waves. Adding the potential of both kinds of waves, owing to the function $\mathrm{e}^{-k z}$ for the dependence of the periodical wave quantities on the depth coordinate $z$, larger (maybe tremendously larger) wave amplitudes are obtained on a crest of the stationary waves than in a stationary wave trough. In principle, this effect would be cancelled approximately by the diffraction potential. However, this would require a very accurate determination of the diffraction potential using the exact free-surface boundary condition; the mirror source method used here to approximate the free-surface boundary condition is not sufficient for this. Therefore, in equation (25) instead of the position vector $\vec{x}$ the modified vector $\left(x, y, z-z_{\mathrm{st}}\right)$ is used, where $z_{\mathrm{st}}$ is the height of the stationary wave along the hull at coordinate $x$. This corresponds to the approximation that, along the ship's deformed waterline, the amplitude of the incoming waves (without diffraction) is the same on crests and in troughs of the stationary wave field.

\section{EXAMPLE}

The method is applied to a container ship described in Table 1. Figure 1 shows the panel grids on hull and free surface used for the steady-flow calculation and the free-surface deformation. For the ship without forward speed, the transom is not immersed, and the waterline is shorter and pointed at the ship's stern. The parts of the hull which are submerged only due to stern wave and squat are responsible for much of the springing excitation, because most of the other hull regions are deeply submerged, so that wave pressure oscillations are extremely small for the short waves responsible for springing excitation. Thus, without stern wave and squat the excitation is grossly underpredicted. 
Table 1 Main particulars of the ship used as example

\begin{tabular}{llll}
\hline$L_{\mathrm{pp}}$ & $304 \mathrm{~m}$ & Draft at AP & $13.69 \mathrm{~m}$ \\
Breadth & $42.8 \mathrm{~m}$ & Draft at FP & $12.56 \mathrm{~m}$ \\
Total mass & $115500 \mathrm{t}$ & Investigated speed & $20 \mathrm{knots}$ \\
\hline
\end{tabular}

Figure 2 shows the deformation contours of the first six approximate modes used for the computation. For comparison, the springing vibration shapes determined at the first two resonance frequencies in head waves are shown also.

Figure 3 illustrates springing excitation generalized force and springing transfer function at the foremost FE node in the neighborhood of the 2-node eigenfrequency for two wave angles. Figure 4 shows corresponding transfer functions around the second vibration eigenfrequency. The excitation does not show the extreme variations between minima and maxima as found, e.g. in reference [2], using strip theory in combination with Newman's reverse-flow theorem [3].

Besides the original panel mesh A (Fig. 1) having 3064 panels on one side of the hull, two coarser meshes B and C consisting of 1522 (B) and 819 triangles (C) were used. They gave slightly different resonance frequencies; for the first bending resonance

$$
\begin{aligned}
& \mathrm{A}: 3.307 \mathrm{rad} / \mathrm{s} \\
& \mathrm{B}: 3.335 \mathrm{rad} / \mathrm{s} \\
& \mathrm{C}: 3.364 \mathrm{rad} / \mathrm{s}
\end{aligned}
$$

However, the excitation differs substantially between these two meshes (Fig. 5). The mesh of intermediate fineness gives the smallest excitation. Thus even the fine mesh may be not fine enough to determine the excitation accurately, and even finer meshes will have to be examined.

From the non-dimensional breadth of the resonance peak $2 \Delta \omega$ at half of the maximum response

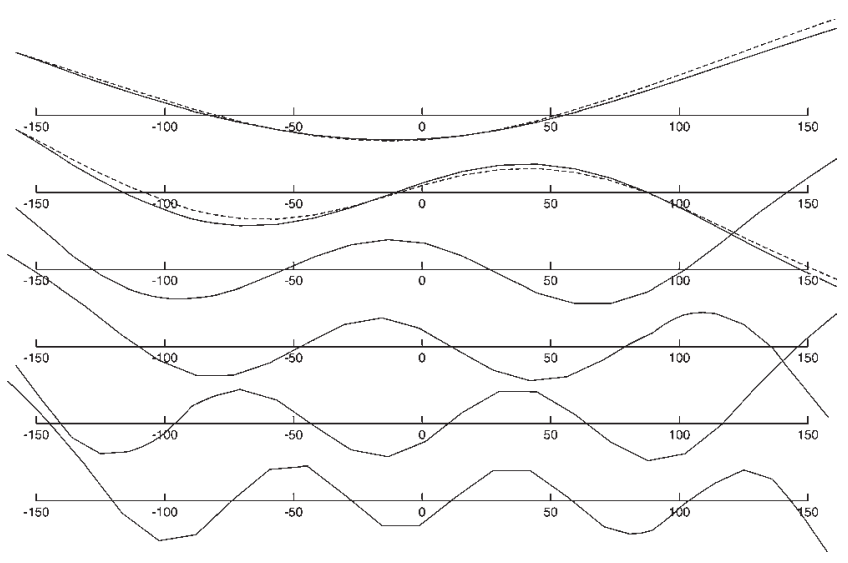

Fig. 2 Mode approximations (full) and deformations at resonance peaks for $\mu=180^{\circ}$ (broken lines)

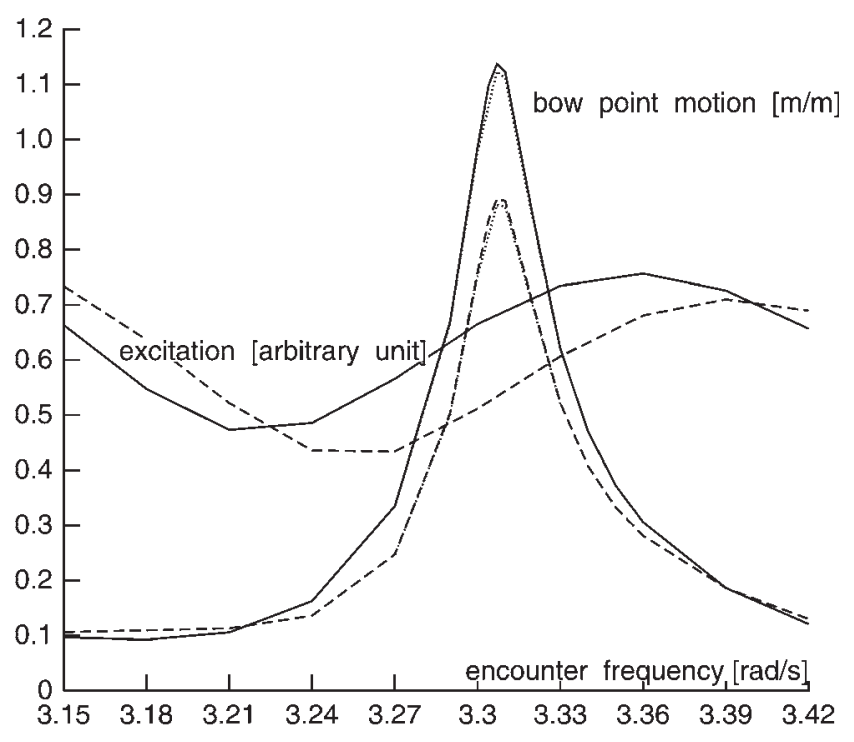

Fig. 3 Springing amplitude of bow point and modal excitation near 2-node vibration resonance for wave directions 160 (broken) and $180^{\circ}$ (continuous lines)

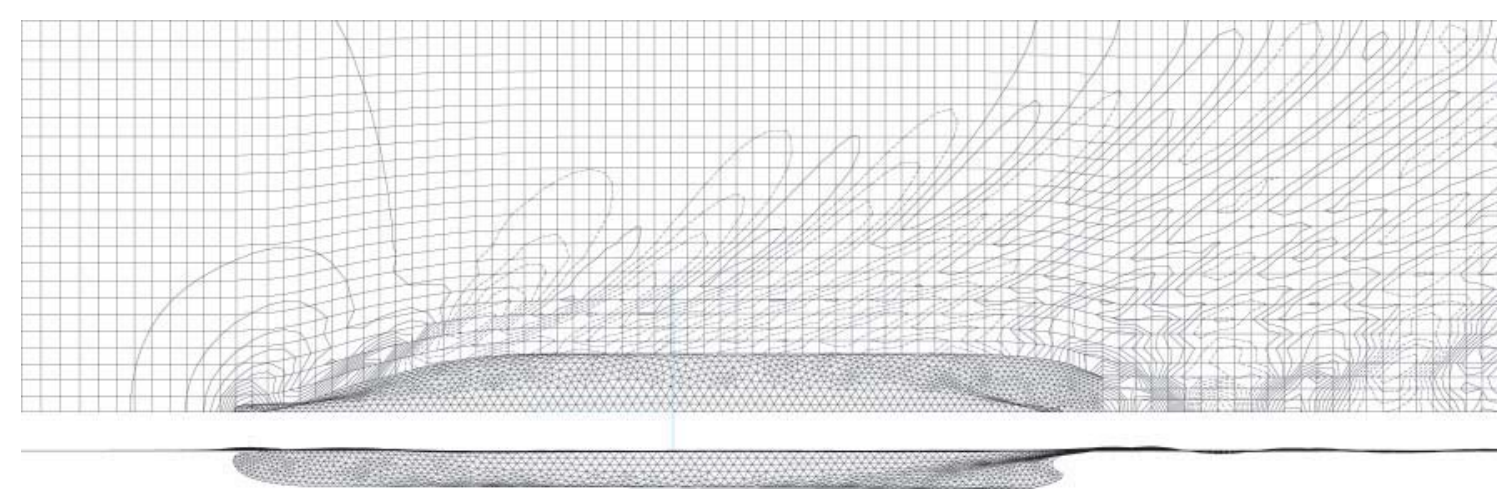

Fig. 1 Panel grids and free surface deformation; height contours in $0.1 \mathrm{~m}$ steps 


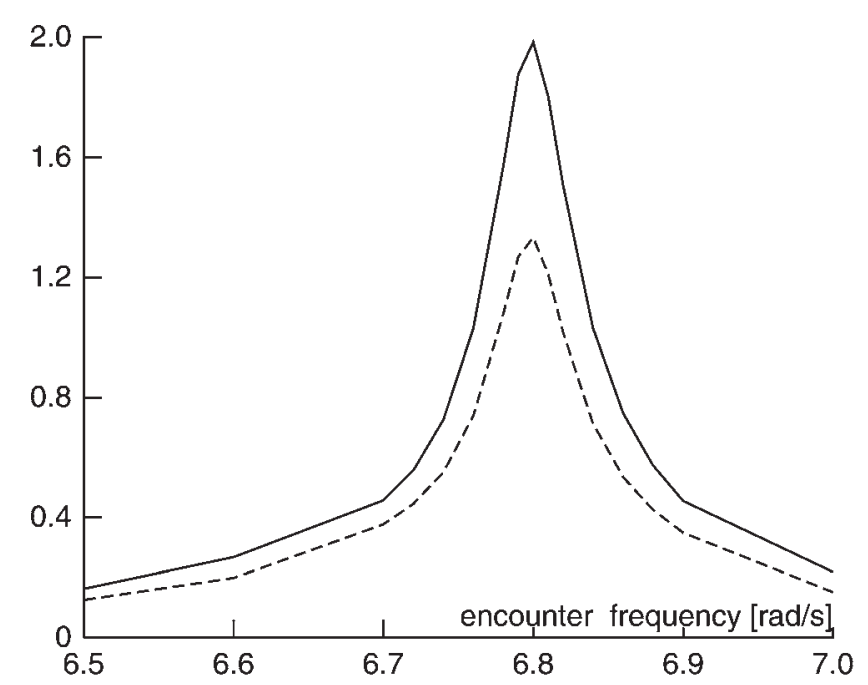

Fig. 4 Springing amplitude of bow point near 3-node vibration resonance for wave directions 160 (broken) and $180^{\circ}$ (continuous lines)

one can estimate the damping ratio $\xi$ (i.e. actual damping/critical damping = logarithmic decrement/ $2 \pi$ ) for the respective mode according to the formula valid for a 1-degree-of-freedom system with small damping

$$
\xi=\frac{\Delta \omega}{\sqrt{3} \omega_{\max }}
$$

The formula gives $\xi=0.41$ per cent for the 2 -node mode (Fig. 3) and $\xi=0.23$ per cent for the 3 -node mode (Fig. 4) for mesh A. This result is unexpected because the theory described in the foregoing, which was used to derive these results, seems not to include any 'net damping'. The imaginary part of $V$ in equation (11) defines damping forces, and it is certainly non-zero. However, it was expected that the imaginary parts of the elements of $V$, which are partly positive, partly negative, would only produce a slight coupling between the approximate modes, but no damping of the really occurring vibrations, because physical processes which extract energy from the vibration are not contained in the theory:

(a) the damping owing to an immersed transom is not covered by the theory because the oscillatory flow was computed without introducing a Kutta condition at the transom and without assuming vortices behind the transom;

(b) the damping owing to wave radiation is not covered because the simplified free-surface condition $\phi=0$ was used;

(c) propeller, bilge keels, etc. were not modelled.

Thus the positive damping found might be ascribed to discretization or rounding errors of the numerical method. To eliminate rounding errors, the programme was alternatively run with double precision variables. However, the differences were tiny at the resonance frequency and negligible otherwise (dotted lines in Fig. 3). To test for discretization errors, damping ratios were determined for the 2node bending mode from results for meshes $\mathrm{A}, \mathrm{B}$, and $\mathrm{C}$. The results were

$$
\begin{aligned}
& \text { A }: \xi=0.41 \text { per cent; } \\
& \text { B }: \xi=0.23 \text { per cent; } \\
& \text { C }: \xi=0.07 \text { per cent. }
\end{aligned}
$$

Discretization errors, however, are expected to be larger for coarser meshes. Thus the damping indicated by the method still needs to be clarified. The larger damping produced by known physical processes, on the other hand, is approximated as explained below and added on the main diagonal of matrix $V$.
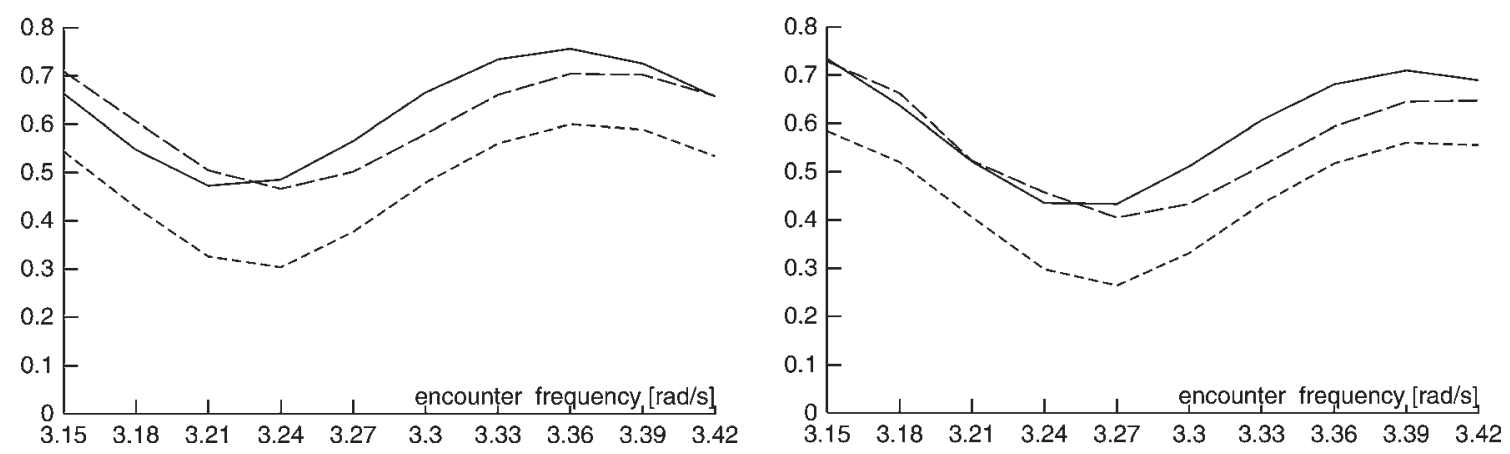

Fig. 5 Excitation (arbitrary unit) of the 2-node bending mode depending on encounter frequency for meshes A (continuous), B (fine broken), and C (coarse broken line) for wave angles $\mu=180^{\circ}$ (left) and $\mu=160^{\circ}$ (right) 


\section{DAMPING}

Vibration damping of ship hull girders is the topic of ship dynamics in which, apparently, the physics is understood least, and erroneous conclusions are published frequently. Most of the relevant publications agree that material (steel) damping is predominant. But listening to the sound of a tuning fork or a piano string demonstrates that the material damping of steel is extremely small if the stress amplitudes are far below the yield stress, as is typical for ship springing. The damping ratio $\xi$ of, e.g., a tuning fork is orders of magnitude lower than the observed damping ratio of the lower vertical vibration modes of ships. Their damping ratios are, typically, in the range of 1 or a few per cent. For instance, Price [9] shows vibratory response measurements of a frigate (p.296) and of the motor vessel 'City of Plymouth' (p.381) after whipping events. The decay of these vibrations indicates damping ratios of about 0.9 per cent and 1.2 per cent, respectively. For the vessel of Table 1, damping ratios between 1.76 per cent and 5.08 per cent were found [10] by using the same method. (On ships without forward speed in sheltered water much smaller damping ratios are found, but these are irrelevant here.) Other processes causing damping of vertical hull-girder vibrations are therefore discussed. For horizontal vibrations, corresponding considerations have not yet been made.

\subsection{Transom effect}

If the transom moves vertically, the fluid around it contains a vertical momentum which remains in the water when the water separates smoothly at the transom contour. The change in periodical vertical momentum between the inflow (in front of the ship, excluding waves) and the outflow behind the vibrating ship corresponds to a vertical oscillatory force distribution on the hull which causes the damping. The vibration energy is transfered to the kinetic energy of the vertical oscillatory fluid motion behind the transom. A strip-theory-like approximation of this force results in the expression for the transom contribution to $\operatorname{Im} V_{j j}$

$$
\operatorname{Im} V_{j j T}=U \omega_{j} w_{j T}^{2} \mu_{z T}
$$

where $U=$ ship speed, $w_{j T}=$ vertical component of $\vec{w}_{j}$ at the transom, $\omega_{j}=$ eigenfrequency of mode $j$, and $\mu_{z T}=2 \mathrm{D}$ added mass of the transom crosssection in vertical motion. For springing frequencies $\mu_{z T}$ is well approximated as

$$
\mu_{z T}=\rho \pi B_{T}^{2} / 8
$$

where $B_{T}$ is the waterline breadth at the transom. Off-diagonal damping terms in $V$ are less important.

Notice that the transom damping is proportional to $U$, and that the factor $B_{T}^{2}$ causes a strong sensitivity to the draft at the aft perpendicular, the stern wave, and to the relative vertical motion between transom and water surface owing to lowfrequency oscillatory waves; the latter will cause variations of the transom damping in the frequency range of the rigid-ship motions.

From equation (46) follows the transom contribution to the damping ratio of mode $j$ as

$$
\xi_{j T}=\frac{\operatorname{Im} V_{j j T}}{2 \omega_{j}^{2} \int_{L} w_{j z}^{2} \mu \mathrm{d} x}
$$

where $\mu$ is the sum of ship mass and added mass for vertical motion, both per length and as functions of $x$. A corresponding equation holds for the other contributions to damping.

\subsection{Wave radiation effect}

Contrary to the $3 \mathrm{D}$ case, in two dimensions the condition (27) (with $U=0$ ) can easily and accurately be satisfied for the frequencies of interest here, for instance by the method [11]. However, an extremely fine discretization is required near the waterline. The resulting vertical damping force amplitude per vertical velocity amplitude and per length can be approximated roughly as

$$
n_{33}=\frac{\rho g \sqrt{A} \tan \alpha}{0.75 \omega}
$$

with $A=$ section area and $\alpha=$ flare angle (between vertical and contour tangent) at the waterline. From $n_{33}$, determined either by the method presented in reference [11] or by equation (49), the wave radiation contribution (index $R$ ) to $\operatorname{Im} V_{j j}$ can be estimated in analogy to the strip theory

$$
\operatorname{Im} V_{j j R}=\omega_{j} \int_{L} w_{j z}^{2} n_{33} \mathrm{~d} x
$$

In the relevant literature the wave radiation effect on damping is held negligible. The reason for this seems to be that $n_{33}$ was computed or estimated for sections with small or zero flare angle, e.g. for Lewis 
sections or other conformal mappings of a semicircle, which all have a flare angle of zero; or that methods or section discretizations were used which are not applicable to the high frequencies of springing.

The formulae given here show that substantial damping is produced only by ship sections which have an extreme flare at the waterline. In merchant ships extreme section flare at the waterline may occur only in front of the transom for a certain range of aft draft. Thus also the wave radiation damping is extremely sensitive to the draft at the aft perpendicular, to the stern wave, and to relative motions between water surface and ship at the stern. Relatively large wave radiation damping occurs if the waterline (including ship waves) is slightly below the transom, but then the transom damping is zero. Thus, depending on the stern wave contour, either transom damping or wave radiation damping can be substantial, but not both at the same time. For waterlines deeply below the transom both kinds of damping are negligible.

\subsection{Bilge keel effect}

In the relevant literature the vibration damping effect of bilge keels is sometimes mentioned, but held to be negligible. The reason for this is, probably, that the interaction between springing and rigidbody ship motions was neglected, which is decisive here.

To estimate the effect roughly, the downward drag force per length, $\delta$, of a ship section moving upward relative to the water is approximated as

$$
\delta=\frac{1}{2} \rho C_{\mathrm{d}} B v_{\text {rel }}^{2}
$$

where $v_{\text {rel }}$ is the time-varying relative motion between the water (if it were undisturbed by the ship) and the ship in the range of the bilge keels. $C_{\mathrm{d}}$ is a resistance coefficient. Only in upward relative motion of the section a broad wake can be produced by flow separation at the bilge keels; thus for downward motion the drag forces are neglected. Notice also that the ship's breadth $B$ appears in equation (51), not twice the height of the bilge keels, because the bilge keels cause a wake the breadth of which is approximately $B$.

The velocity $v_{\text {rel }}$ is the sum of a larger, slowly varying contribution $v_{\text {rell }}$ owing to ship heaving, pitching and wave orbital motion, and a small contribution $v_{\text {rel2 }}$ due to springing. Inserting this sum into equation (51) gives

$$
\delta=\frac{1}{2} \rho C_{\mathrm{d}} B\left(v_{\mathrm{rel} 1}^{2}+2 v_{\mathrm{rel} 1} v_{\mathrm{rel} 2}+v_{\mathrm{rel} 2}^{2}\right)
$$

Only the middle term between parentheses produces a substantial force in phase with the vibration velocity, thus contributing to vibration damping

$$
\delta=\rho C_{\mathrm{d}} B v_{\text {rel1 }} v_{\text {rel2 }}
$$

The ratio $\delta / v_{\text {rel2 }}=$ force per length and per vibration velocity corresponds to the damping coefficient $n_{33}$ of the previous subsection. Thus, in analogy to equation (50), the bilge keel effect on $\operatorname{Im} V_{j j}$ is

$$
\operatorname{Im} V_{j j B}=\omega_{j} \int_{B} w_{j z}^{2} \rho C_{\mathrm{d}} B v_{\text {rel1 }} \mathrm{d} x
$$

Here $\int_{B}$ means integration over the length range of the bilge keels. In estimating $C_{\mathrm{d}}$, one has to take into account that the resistance in oscillatory flow (in the frequency range of the rigid-body ship motions) will be smaller than in stationary flow; thus a value of 0.8 may be assumed tentatively until further information is available. Owing to the slow frequency change of $v_{\text {rell }}$, the bilge keel vibration damping depends on time. If $v_{\text {rell }}$ is a Gauss process with variance $v$, the time-averaged value of $\left|v_{\text {rell }}\right|$ is $0.8 \sqrt{v}$. However, because substantial forces are held to occur only in upward motion, a value of $0.4 \sqrt{v}$ has to be used in equation (54) to obtain the time-averaged damping.

\subsection{Propeller effect}

In reference [12] the following expression for the damping force of a screw propeller in transverse or vertical vibration is given

$$
n_{33 P}=0.248 \rho n_{P} D P^{2} A_{E} / A_{0}
$$

where $n_{P}$ is number of revolutions per time, $D$ diameter, $P$ pitch and $A_{E} / A_{0}$ the expanded blade area ratio of the propeller. The formula is based on lifting-line computations. Nearly the same formula (without $A_{E} / A_{0}$ and 0.25 instead of 0.248 ) is given in reference [6], however based on measurements [13] of the stationary force on a propeller in oblique flow.

A further test of the validity of equation (55) is made by using results of attentive RANSE calculations [14]. For a model propeller $(D=0.25 \mathrm{~m}, P /$ $D=0.959, A_{E} / A_{0}=0.588$ ) in oblique flow of various 
angles $\gamma$ at $J=0.53$ the time-averaged transverse force coefficient was determined as

$$
\bar{K}_{y}=\frac{\bar{Y}}{\rho n_{P}^{2} D^{4}}=0.09 \gamma
$$

On the other hand, formula (55) gives for this propeller model, using $\gamma=v_{y} / V_{A}$ and $\bar{Y}=n_{33 P} v_{y}$

$$
\bar{K}_{y}=0.248(P / D)^{2} J\left(A_{E} / A_{0}\right) \gamma=0.071 \gamma
$$

The coincidence appears reasonable.

A formula analogous to equation (50) gives the propeller contribution to $\operatorname{Im} V_{j j}$

$$
\operatorname{Im} V_{j j P}=\omega_{j} w_{j z P}^{2} n_{33 P}
$$

where $w_{j z P}$ is the $z$ component of the eigenform $w_{j}$ at the location of the propeller.

The influence of the tilt of the propeller owing to the $w_{j z P}^{\prime}$ is, especially for the 2-node vibration mode, much smaller than that due to $w_{j z P}$ and is omitted here.

\subsection{Effect of hatch cover friction}

In ships carrying containers on the hatch covers, the friction between hatch covers and coamings produces vibration damping which may be worthwhile discussing here. Substantial motion between hatch cover and coaming can appear if the stoppers, which fix some points of the cover relative to the coaming, and their clearings are designed to avoid that substantial longitudinal stresses are transferred from the hull to the hatch cover. Such a construction seems to be applied in most cases [15].

If there were only hull deformations due to springing, the friction between hatch coaming and cover would eliminate relative motions between cover and coaming for small vibration amplitudes. On the other hand, for very large amplitudes the relative motion amplitude would approximate the value

$$
\Delta x=\varepsilon l
$$

where $\varepsilon$ is the longitudinal strain amplitude of the hatch coaming and $l$ the hatch length. For the modal vibration $j$ the strain amplitude is, according to the beam approximation

$$
\varepsilon=\chi_{j}^{\prime}\left(z_{H}-z_{0}\right)
$$

where $z_{H}$ and $z_{0}$ are the $z$ coordinates of the hatch coaming and of the beam axis, respectively, and $\chi_{j}^{\prime}$ is the $x$ derivative of the the rotation angle of the beam cross-sections in mode $j$ for motion amplitude $\hat{v}_{j}=1$. Owing to shear lag, actual strains in the hatch coaming will be somewhat smaller.

If substantial low-frequency wave strains are superimposed to the springing strains, relative motions in phase with the vibration will occur even in case of small springing amplitudes. Thus the relative motion between cover and coaming depends on the amplitudes of springing strain, of the low-frequency strain amplitude, the cover loading $g m_{H}$ where $m_{H}$ is the mass stowed on the cover, and on the friction coefficient $\mu$, for which different authors give values between 0.15 and $0.6[\mathbf{1 5}]$. To obtain a rough estimate of the hatch cover damping, a reduction factor $r$ with $0 \leqslant r \leqslant 1$ is applied in equation (59)

$$
\Delta x=r \varepsilon l
$$

From the definition of matrix $V$ it can be deduced that the time-averaged damping power due to an element $V_{j j}$ on the main diagonal of $V$ is, for a modal vibration with $\hat{v}_{j}=1$

$$
P=\frac{1}{2} \omega_{j} \operatorname{Im}\left(V_{j j}\right)
$$

On the other hand, the time-averaged damping power due to hatch cover friction is

$$
P=\frac{2 r|\varepsilon| l \mu g m_{H}}{2 \pi / \omega_{j}}
$$

Combining equations (62) with (63) and using equation (60) gives the hatch friction contribution to $\operatorname{Im} V_{j j}$ as

$$
\operatorname{Im} V_{j j H}=\sum_{\text {all hatches }} \frac{2}{\pi} r\left|\chi_{j}^{\prime}\left(z_{H}-z_{0}\right)\right| l \mu g m_{H}
$$

\subsection{Other causes of vibration damping}

Depending on the case, noticeable springing damping may be produced by the cargo of certain ships, especially by cars (in ferries and car carriers) and possibly by certain kinds of bulk cargo.

\subsection{Numerical results}

For the ship of Table 1 the above approximate damping formulae were applied, using the following data (most of them estimated) 
Table 2 Damping ratios $\xi$ in per cent for the ship of Table 1 owing to different damping processes for 2-node until 7-node bending modes

\begin{tabular}{|c|c|c|c|c|c|c|}
\hline $\begin{array}{l}\text { Number of nodes } \\
\text { Approximate eigenfrequency }(\mathrm{Hz})\end{array}$ & $\begin{array}{l}2 \\
0.505\end{array}$ & $\begin{array}{l}3 \\
1.040\end{array}$ & $\begin{array}{l}4 \\
1.578\end{array}$ & $\begin{array}{l}5 \\
2.067\end{array}$ & $\begin{array}{l}6 \\
2.636\end{array}$ & $\begin{array}{l}7 \\
3.061\end{array}$ \\
\hline $\begin{array}{l}\text { Transom } \\
\text { Wave radiation } \\
\text { Bilge keels } \\
\text { Propeller } \\
\text { Hatch cover friction }\end{array}$ & $\begin{array}{l}1.850 \\
0.400 \\
0.080 \\
0.030 \\
0.006\end{array}$ & $\begin{array}{l}0.910 \\
0.075 \\
0.034 \\
0.022 \\
0.003\end{array}$ & $\begin{array}{l}0.580 \\
0.028 \\
0.027 \\
0.006 \\
0.002\end{array}$ & $\begin{array}{l}0.580 \\
0.016 \\
0.021 \\
0.004 \\
0.001\end{array}$ & $\begin{array}{l}0.300 \\
0.007 \\
0.022 \\
0.001 \\
0.001\end{array}$ & $\begin{array}{l}0.210 \\
0.004 \\
0.016 \\
0.001 \\
0.001\end{array}$ \\
\hline Sum & 2.366 & 1.044 & 0.643 & 0.622 & 0.331 & 0.232 \\
\hline
\end{tabular}

1. For the propeller: diameter $8 \mathrm{~m}$, pitch $6.8 \mathrm{~m}$, and advance coefficient 0.54 .

2. For the bilge keels: $C_{\mathrm{d}}=0.8$, time-average of $\left|v_{\text {rel }}\right|=0.75 \mathrm{~m} / \mathrm{s}$, and bilge keel length $86 \mathrm{~m}$.

3. For the hatches: number 20, length $12.3 \mathrm{~m}, r=0.3$, $\mu=0.25, z_{h}-z_{0}=18 \mathrm{~m}$, and hatch load within each bay $1500 \mathrm{t}$ for the aft and middle hatches, less for the forward hatches; total hatch load $28000 \mathrm{t}$.

These data produced the damping ratios of Table 2. It shows that, in this example, the transom damping is dominant, followed by the wave radiation damping. Bilge keel, propeller and hatch cover damping are negligible here. In other cases, however, especially for ships without an immersed transom in a heavy seaway, bilge keel and propeller damping were found noticeable.

The total damping of the 2-node vibration is in the lower range of damping values given in reference [10] for the same ship.

\section{ACKNOWLEDGEMENTS}

The support of Dr V Shigunov and his colleagues from Germanischer Lloyd, who provided the ship and measurement data in section 11 is gratefully acknowledged.

(c) Author 2009

\section{REFERENCES}

1 Grim, O. Elastic ship vibrations excited by nonlinear forces in a natural seaway, report 325. Institut für Schiffbau, Hamburg, 1975. In German.

2 Söding, H. Springing of ships, report 7, Institute for Ship Design and Ship Theory, Hannover, 1975.

3 Newman, J. N. The exciting forces on a moving body in waves. J. Ship Res., 1965, 9, 190-199.

4 Oberhagemann, J., El Moctar, O., and Schellin, T. E. Fluid-structure coupling to assess whipping effects on global loads of a large containership. 27th Symposium on Naval hydrodynamics, 2008.
5 Söding, H. A method for accurate force calculations in potential flow. Ship. Technol. Res., 1993, 40(4), 176-186.

6 Jensen, G. and Söding, H. Ship vibrations, Lecture Notes, 2003. In German.

7 Söding, H. and Conrad, F. Analysis of overtaking manoeuvres in a narrow waterway. Ship Technol. Res., 2005, 52(4), 189-193.

8 Söding, H. and Bertram, V. A 3-d Rankine source seakeeping method. Ship Technol. Res., 2009, 56(2), 50-68.

9 Price, W. G. (ed.) The dynamics of ships, 1991 (The Royal Society, London).

10 Kaufmann, J. and Shigunov, V. Know-how springing, Germanischer Lloyd, Report No. 2009.035, 2009.

11 Bertram, V. and Söding, H. Verification of the PDSTRIP 2-d radiation problem module, NuTTS, 2007.

12 Schwaneke, H. Considerations about the problem of hydrodynamically excited vibrations of the propeller and the shafting (in German). Proc. German Ship Tech. Soc. (STG), 1963, 252-280.

13 Meyne, K. and Nolte, A. Experimental investigation of hydrodynamic force and moment on a propeller blade in oblique flow (in German). Schiff und Hafen, 1969.

14 El Moctar, O. Numerical computations of flow forces in ship manoeuvring. Ship Technol. Res., 2001, 48(3), 98-123.

15 Rodionov, A. N. Some aspects of interaction between the hull and hatch covers of containerships. Ship Technol. Res., 1993, 40, 59-70.

\section{APPENDIX}

\section{Notation}

A

$A_{E}$

$A_{\mathrm{S}}$

$A_{0}$

$B$

$C_{\mathrm{d}}$

$D$

$D$ hull section area

expanded blade area of propeller

shear area of cross-section

area of propeller circle

breadth of waterline

resistance coefficient

$=\left(\boldsymbol{w}_{k}^{T} \mathbf{D}_{j} \boldsymbol{w}_{j}, k=1 \ldots J, j=1 \ldots J\right)$

propeller diameter 
$\mathcal{D}$

D

E

$\hat{\boldsymbol{F}}$

$\hat{F}$

$\mathcal{F}$

$g$

G

$\overrightarrow{\boldsymbol{G}}$

$i$

I

$\boldsymbol{w}_{j}$ damping operator

damping matrix

Young's modulus

FE excitation vector

$=\left(\mathbf{w}_{k}^{T} \hat{\boldsymbol{F}}, k=1 \ldots J\right)$

excitation

gravity acceleration

shear modulus

weight force

imaginary unit

cross-section moment of inertia

around $y$-axis

number of modes taken into account

propeller advance ratio

$=\left(\mathbf{w}_{k}^{T} \mathbf{K}_{j} \mathbf{w}_{j}, k=1 \ldots J, j=1 \ldots J\right)$

stiffness matrix

stiffness operator

hatch length

length of finite element

mass stowed on hatch cover

$=\left(\mathbf{w}_{k}^{T} \mathbf{M}_{j} \mathbf{w}_{j}, k=1 \ldots J, j=1 \ldots J\right)$

mass matrix

mass operator

propeller number of revolutions per time

damping force per length for vertical motion

normal vector on hull; time average

normal vector on hull; instantaneous

pressure

propeller pitch

reduction factor

wetted hull surface

time

vibrational translation

FE node translation vector

ship speed

mode superposition factor

relative motion between water and

bilge keel

virtual work

$=-\left(K+i \omega_{\mathrm{e}} D-\omega_{\mathrm{e}}^{2} M\right)$

$=\left(-\mathcal{K}+i \omega_{\mathrm{e}} \mathcal{D}-\omega_{\mathrm{e}}^{2} \mathcal{M}\right)$

FE node translations and section

rotations of mode $j$ $x, y, z$

$\vec{x}$

$\bar{Y}$

$z_{0}$

$\alpha$

$\vec{\alpha}$

$\delta$

$\varepsilon$

$\mu$

$\mu$

$\mu$

$\bar{\mu}$

$\mu_{z}$

$\vec{v}$

$\rho$

$\phi$

$\vec{\chi}$

$\omega_{\mathrm{e}}$

^

Lower indices

$B$

$H$

$j$

$k$

$l$

$P$

$T$

W

$P$

$R$

$S$

Upper indices

$d$

$w$

(0)
Cartesian coordinates directed forward, to starboard, downward position vector average transverse propeller force $z$ coordinate of beam axis

flare angle at waterline rotation of hull normal vertical damping force per length strain in coaming friction coefficient solid mass per length wave angle solid mass moment of inertia per length added mass per length of a ship section in vertical motion wave vector $\mathrm{ik}(-\cos \mu, \sin \mu$, i) damping ratio water density flow potential rotation vector of cross-sections wave encounter frequency designates complex amplitudes

bilge keel

hatch coaming

mode index

mode index

index of mass item

pressure

transom

weight

propeller

wave radiation

structure

diffraction

wave

steady flow owing to ship speed 